\title{
Decision-Making and Depressive Symptomatology
}

\author{
Yan Leykin • Carolyn Sewell Roberts • \\ Robert J. DeRubeis
}

Published online: 4 May 2010

(C) The Author(s) 2010. This article is published with open access at Springerlink.com

\begin{abstract}
Difficulty making decisions is a core symptom of depressive illness, but the nature of these difficulties has not been well characterized. The two studies presented herein use the same hypothetical scenarios that call for a decision. In Study 1, participants were asked to make and explain their decisions in a free-response format, as well as to describe their prior experiences with similar situations. The results suggest that those with more depressive symptoms make decisions that are less likely to further their interests. We also identified several interesting associations between features of decision-making and the presence of depressive symptoms. In Study 2, participants were guided through their decisions with simple decision tools to investigate whether the association between depressive symptoms and poor decisions is better accounted for by failure to use of good decision-making strategies, or by other factors, such as differences in priorities or goals. With this minimal intervention the quality of decisions no longer declined significantly as a function of depressive symptom severity. Moreover, few associations between depressive symptom severity and decision-related goals and priorities were evident, suggesting that the previously-exposed difficulties of depressed individuals with
\end{abstract}

\footnotetext{
Y. Leykin $(\bowtie)$

Department of Psychiatry, University of California,

San Francisco, 3333 California St., Suite 465,

San Francisco, CA 94143-0848, USA

e-mail: Yan.Leykin@ucsf.edu

C. S. Roberts

Bryn Mawr College, Bryn Mawr, PA, USA

R. J. DeRubeis

University of Pennsylvania, Philadelphia, PA, USA
}

decision-making were largely the result of their failure to use effective decision-making techniques.

Keywords Depression · Decision-making ·

Decision quality $\cdot$ Cognitive therapy

\section{Introduction}

Depressed individuals have difficulties making decisions (e.g., Klerman 1980; Nezu and Perri 1989). Indecisiveness is a symptom common enough in depressed individuals to have been included in the criteria for the disorder (APA 2000). The choices made by depressed persons may also be sub-optimal. Studies have shown that depressed, relative to non-depressed persons, make qualitatively different decisions (Chambers et al. 1996; Kulin et al. 1998; Suri et al. 2004), leading many doctors and psychotherapists to suggest to their patients that they should avoid making major life choices while in a depressed state.

Good decision-making happens when rational thinking is followed by the enactment of a decision that maximizes utility. Rational thinking involves the ability to understand and evaluate alternatives, to make judgments that are relatively free of biases, and appropriately appraise the consequences of decisions. Compared to other possible outcomes, the outcomes of the most productive decisions have the highest utility (subjective value, or goodness, e.g., Bell et al. 1988) to the decision-maker. However, because utility judgments can be influenced by factors such as strong emotions or incorrect predictions of future preferences (Loewenstein et al. 2003), making the most productive choice is often difficult. Biased utility judgments may lead to the selection of a suboptimal alternative and the failure to maximize experienced utility (Kahneman et al. 1997). 
The pessimistic thinking style of depressed individuals may result in a heightened sense of potential disappointment in the expected outcome. The expectations of negative emotions such as regret and disappointment may carry a significant disutility (Bell 1982; Loomes and Sudgen 1982). A tendency to anticipate disappointment may result in choices that appear to the decision-maker to be optimal at the time they are made but which will, over time, prove not to be in the person's ultimate best interest. For example, a person may turn down a promotion due to an overriding concern that one is incapable of handling the responsibility, the expectation of disappointment at the possible subsequent demotion, or simply a "bad feeling" about the situation. A good decision is thus the one that has the most "lifetime" utility, that is, the one that provides the most good for the person over time.

There are several lines of theory and research that suggest that depression is associated with maladaptive decision making. The "portfolio theory" proposed by Leahy (1999, 2000, 2001, 2002) represents a broad description of psychopathological decision-making. Borrowing from Economics, the portfolio theory focuses on the "individual's perception of his or her resources, ability to produce future resources, diversification, emphasis on maximization of rewards or minimization of costs, potential for regret, hedonic utility for gains and losses, and risktolerance" (Leahy 2001, p. 343). Leahy has found that depressed persons maintain a negatively biased portfolio, with beliefs that they possess few assets and low future potential, and that they use a risk-averse approach in which expected losses are minimized at the expense of potential pleasure from possible gains.

A number of psychological factors that affect decisionmaking have also been found to be altered in those with depressive symptoms. For instance, risk aversion was demonstrated in depressed individuals by Murphy et al. (2001). Participants engaged in a task that required them to select a "winning" box, with known probability of wins per box, and to bet a limited amount of "money" on a box they expected to win. Although depressed subjects were as likely as the controls to choose the winning boxes, they took longer to decide on a winning box, and won less money due to a tendency to increase their bets at a lower rate, compared to controls. Depressed subjects were thus more cautious in their decisions, and therefore took fewer risks.

Listlessness and passivity, symptoms frequently observed in depression, are likely to affect decision-making. Depressed individuals also commonly exhibit hopelessness (Abramson et al. 1989) and helplessness (Abramson et al. 1978), which likely leads to a lower likelihood of approaching decisions actively, because expending effort would be seen as useless. Anhedonia and decisional passivity may also reinforce one another. Decisional passivity is likely to cause a variety of negative outcomes; the accumulation of such outcomes can lead to reduced expectation of positive events or pleasure.

Findings from studies of decisions and regret have shown that depressed individuals are especially likely to regret their decisions (Monroe et al. 2005; Schwartz et al. 2002), a tendency that may also contribute to decisional passivity. Anticipatory regret likely serves as a warning mechanism, protecting a decision-maker from bad decisions and prompting him or her to re-evaluate possible alternatives. Inappropriate or excessive regret can thereby impair future decision-making. Given the common tendency of people to experience more regret for active, rather than passive, choices (Ritov and Baron 1995), anticipatory regret may bias a person towards inaction. People may believe, irrationally, that by accepting a default choice passively, they are avoiding making a decision and thereby minimizing their responsibility for the outcomes of that choice.

Although some aspects of depressed persons' decisionmaking processes have been shown to differ from those of the nondepressed, little research has been directed at the important related question of whether depressed individuals make good decisions, that is, decisions that ultimately serve their best interests. The purpose of Study 1 was to determine whether depressive symptoms are associated with the quality, or productivity, of decisions, as well as with features of decision-making processes that can affect the quality of decisions. Extrapolating from the research cited thus far, some of these features, such as tolerance for uncertainty or ambiguity, risk-seeking, perception of resources, or passivity, are expected to be affected by depression and to influence decision making. Other features, such as analytical thinking (employment of good decision-making practices) or information gathering, are known to impact decision-making; whether they are associated with depressive symptoms is not yet well understood.

Of specific interest are the decisions depressed people make in their own lives, as well as consequences of these decisions. Because depressed individuals may decide differently when faced with an abstract, as opposed to a reallife, task (Young and Bentall 1995), and to enhance the ecological validity of the study, hypothetical real-life situations that call for a decision were used in this study. Hypothetical scenarios from the Means-Ends Problem Solving test (MEPS; Platt and Spivack 1975) have previously been used in research on depressed individuals, and difficulties with their problem-solving process were reported (Marx et al. 1992; Wierzbicki 1984; though see also Doerfler et al. 1984, and Joffe et al. 1990). Unlike the MEPS test, on which participants are asked to arrive at an outcome specified by the test, the scenarios used in this 
study were open-ended. Participants were therefore free to generate their own goals and approaches.

\section{Study 1}

Methods

\section{Participants}

One hundred and twenty-five participants were recruited on the Internet from depression-related discussion groups and forums, and from a subject pool of participants in per-pay Web-based studies. Participants were mostly female $(64.8 \%)$, and their average age was $36.2(\mathrm{SD}=11.2)$. Almost two-thirds of the participants $(64.8 \%)$ reported having been given a diagnosis of depression in the past, $39.2 \%$ were currently taking antidepressant medications, and $28.0 \%$ were currently seeing a therapist for their depression.

\section{Materials}

The Beck Depression Inventory-II (BDI; Beck et al. 1996) is a standard and widely-used 21-item self-report measure of depressive symptoms. Question 9, which assesses for suicide risk, was removed from the scale in compliance with the suggestion of the University of Pennsylvania Institutional Review Board. This limited the possible range of the BDI to 0-60. The average BDI score in the sample was $23.3(\mathrm{SD}=16.1$; range $=0-56)$.

The Decisions and Reasons Questionnaire, developed for this study, consisted of six short scenarios, covering social, career-related, potential conflict, self-improvement, family, and relationship situations, each of which calls for a decision. For each scenario, participants were asked to write (free response) their choice of action and the reason(s) for the choice. Then, they were asked to indicate whether they had any past experiences in similar situations. If a past experience was noted, follow-up questions assessed whether the decision that was made in the prior situation was the same or different from the one indicated for the hypothetical scenario. Lastly, participants described, in a free-response mode, the outcome of the past decision.

A coding system was applied to the free-response answers of the Decisions and Reasons Questionnaire. Responses were scored by two Master's level raters (Y.L. and C.S.R.) blind to the participant's depression score or current treatment status. Responses were scored based on:

- The manualized scoring system

- The participant's idiosyncratic responses, such that the score could be adjusted to reflect the participant's unique situation.
The scoring approach was modeled on the Ways of Responding questionnaire (Barber and DeRubeis 1989). As the free responses varied widely in content, not every dimension could be rated for each response. Thus, a twostep process was applied for each response: one rater identified scorable dimensions, then both raters scored the identified dimensions, and the two scores were averaged. For each scenario, the decision and the reasons for that decision were rated on 11 dimensions described below, on a 5-point Likert scale. Dimensions that were selected were deemed to be both relevant to decision-making and likely to be influenced by depressive symptoms. The dimensions were:

1. Productivity - the evaluator's rating of the likelihood that the outcome of the decision would ultimately be in the person's best interest. Although the scoring was manualized, for productivity ratings the evaluators were particularly mindful of the participant's unique situation. For instance, in general, attending a party with a mindset to enjoy oneself and to engage actively with other guests (Scenario 3) would be considered an optimal response. However, if a participant reports that s/he consistently has severe panic attacks every time s/he goes out in public, a polite refusal might be a better choice, unless s/he chooses to attend while undergoing panic disorder treatment (even of the self-initiated variety) for the purpose of a therapy homework experiment and/or exposure.

2. Activity required-the degree of effort a person could be expected to expend as a result of the decision.

3. Risk seeking - the amount of risk, stated or implied, potentially involved in the decision.

4. Resolution of ambiguity - the likelihood that the decision will resolve an apparent ambiguity.

5. Information gathering-the degree of the desire, stated or implied, for the pursuit of additional information.

6. Existing resources - the perception of the availability of resources, including assistance from other people or the respondents' own talents.

7. Aggression vs. Prosociality-an expression of, or desire for, either good will or ill will towards others.

8. Optimism-the degree of positivity regarding expected future events or outcomes.

9. Positive self-reference-the degree of positivity in self-referring statements.

10. Analytical thinking - an indication of the use of adaptive thinking strategies, e.g., evaluation of all options, following useful decision-making rules, thinking rationally. 
11. Emotionality - the negativity or positivity of the emotional tone apparent in the response.

Productivity, Activity Required, and Analytical thinking were rated most frequently, as virtually every response could be judged on these dimensions. Reliability for the identification of a response as ratable or not ratable on a given dimension was high (Cohen's kappa coefficients $=0.85$ ). Intra-class correlations were calculated to estimate reliabilities for scores assigned to ratable dimensions; these ranged from 0.78 to 0.96 , indicative of good-to-excellent reliability. To create composite dimension scores, nonmissing scores were averaged across all scenarios for each dimension. The correlations between pairs of decisionmaking process variables (\#2 - \#11 above) ranged from negligible (10.01I) to high $(|0.81|)$, with a median of $10.32 \mid$. Across all six scenarios, most respondents (from a low of $63 \%$ on the least ratable dimension to over $99 \%$ on the four most ratable dimensions, median $=91 \%$ ) provided responses that could be rated. For each individual scenario, the percentages of responses that could be rated were lower (from $17 \%$ on the least ratable dimension to $97 \%$ on the two most ratable; median $=32 \%$ ), as expected.

The responses regarding the outcomes of decisions made in real-life situations that resembled the decision scenarios were rated on a 3 -point Likert scale ( $1=$ Negative, $2=$ Neutral, 3 = Positive) by both raters, averaged between evaluators, and averaged across the six scenarios. The ICC of the rating of the outcomes of past decisions was 0.83 . All reliabilities were estimated based on two raters, both of whom rated protocols from the first 74 participants.

\section{Results}

\section{Relationship to Depression}

BDI scores were strongly and significantly correlated with ratings of reduced activity $(r=-0.52, \quad P<0.001)$, reduced perception of existing resources $(r=-0.47$, $P<0.001)$, lower optimism $(r=-0.58, \quad P<0.001)$, lower self-appraisal $(r=-0.28, P<0.01)$, and higher negative emotionality $(r=-0.35, P<0.005)$. BDI scores were also significantly related to lower risk-seeking ( $r=$ $-0.23, P<0.02$ ), lower likelihood of ambiguity resolution $(r=-0.30, P<0.001)$, reduced information gathering $(r=-0.44, \quad P<0.001)$, and lower prosociality $(r=$ $-0.33, P<0.001)$. Importantly, BDI scores were also strongly associated with lower productivity of decisions $(r=-0.53, P<0.001)$ and poorer analytical thinking ( $r=-0.48, P<0.001$ ), indicating that individuals with greater depressive symptomatology appeared to be making their decisions in either a sub-optimal or maladaptive fashion, resulting in poorer decisions. All but two of the above correlations remained significant when controlling for those demographic (age, gender) and/or current treatment (current antidepressant use, current psychotherapy) variables that were significantly related to the respective dimension. The associations between the BDI and Selfappraisal $(r=-0.17, P<0.14)$ and Risk-seeking $(r=$ $-0.16, P<0.12$ ) became nonsignificant.

Because a sub-optimal approach to decisions is likely to impact the productivity of decisions negatively, we conducted tests to determine whether the data were consistent with a hypothesis that Analytical thinking scores mediate the relationship between BDI scores and productivity scores. Analytical thinking scores were entered into a regression model that predicted productivity from BDI scores. Although the relationship of BDI to productivity remained significant $(t(118)=-2.99, P<0.005)$, the unstandardized beta was reduced from $\beta=-0.03$ to $\beta=-0.01$, indicative of partial mediation. A Sobel test confirmed the significance of the test for mediation $(Z=$ $-5.35, P<.001)$.

\section{Past Decisions}

A strong association between BDI scores and ratings of past outcomes was obtained, such that participants with higher BDI scores also reported poorer outcomes of their past decisions $(\beta=-.02, t(120)=-5.47, P<0.001)$, even when we controlled statistically for the number of scenarios about which participants reported past outcomes. Interestingly, when facing a similar current problem, individuals with higher BDI scores more often chose decisions that had previously resulted in negative outcomes $(\beta=.01, t(120)=2.72, P<0.01)$, even when we controlled for the proportion of past outcomes they reported to have been negative. This provides additional evidence for the reduced capacity to evaluate one's options carefully, and, perhaps, reduced ability to learn from one's prior decision-making experiences.

\section{Discussion}

The purpose of this study was to characterize the decisionmaking processes that are related to depressive symptom severity. Several interesting relationships between depressive symptoms and features of decision-making were revealed. Some of the observed relationships could be anticipated given the features and deficits that have been documented in depression, including the pessimistic outlook on life and a predominance of negative emotions (e.g., Abramson et al. 1978, 1989). In our study, both pessimism and negative emotionality were correlated with the depression symptoms. As predicted by Leahy's Portfolio model, individuals with a greater level of depression 
symptoms were also found to perceive fewer resources as being available to them, though risk-aversion, another facet of the model, was not a strong correlate of depressive symptoms. Similarly, the tendency of depressed persons to be unwilling to take an active approach to decisions might be predicted given the lack on energy and reduced motivation frequently observed in depressed individuals.

Some of the other observed relationships were not directly predictable from what is known about depressed individuals, and may therefore provide new insights into the deficits in decision-making of depressed individuals. Most important among these were the strong negative associations found between depressive symptoms and the productivity of decisions, as well as between depression and the quality of the decision making process (i.e., degree of analytic thinking). Thus, individuals with greater depressive symptomatology used fewer adaptive strategies in making their decisions and, likely because of that, decisions they made were likely to be less productive. These findings were further supported by the associations between depressive symptoms and reduced search for information as well as a decreased likelihood that a decision will resolve ambiguities or uncertainties.

The inference that decision-making processes of depressed individuals are maladaptive is reinforced by the apparent readiness of depressed persons to choose an alternative that has already failed them at least once. A related finding - that depressed individuals reported worse outcomes of past decisions - may be explained by the general tendency to evaluate situations in a negative, pessimistic manner. However, repeating a strategy that had failed in the past cannot be similarly explained. Even if the past outcome was objectively better than was perceived by the depressed individual, inasmuch as its perception was negative, this should be expected to inform subsequent decisions. Instead, the reverse was true for those with greater depression scores. Naturally, making the same decision as a previously failed one may be warranted in some situations, for example if circumstances have changed, however, there is no good reason why this would be more likely to occur with individuals who have more depressive symptoms.

\section{Study 2}

The results of the Study 1 suggest that individuals with greater depressive symptoms make less productive decisions and engage fewer adaptive decision-making strategies. A possible conclusion, especially in light of results from the meditation analysis, is that a general failure to use good strategies was chiefly responsible for the poor quality of the decisions. Alternatives include the possibilities that depressed individuals judge the expected utility of outcomes differently from nondepressed, or that they have different goals for their decisions. If depressed individuals value different outcomes, their decisions would be different from those of non-depressed persons, even if they use good decision-making strategies.

Study 2 was undertaken to evaluate the outcomes of the decisions separate from the use of good decision-making strategies, using a quasi-experimental rather than a statistical approach. The same hypothetical scenarios presented in Study 1 were used to elicit decisions from participants. However, key aspects of the participants' decision-making process were controlled via instructions that guided them through the steps of deciding, thus ensuring that all participants used the same decision-making tools. Observing, once again, a strong relationship between the level of depressive symptoms and the productivity of decisions would suggest that the poorer choices of depressed persons are due to aspects of decision-making other than poor decision strategies. Alternatively, if no relationship is found between depressive symptoms and productivity of decisions, the hypothesis that the lack of sound decision-making strategies is responsible for the poor decisions of those with more depressive symptoms would receive support. As a further test of these hypotheses, we evaluated whether individual differences in decision-relevant goals could be explained by variations in depressive symptom levels.

\section{Methods}

\section{Participants}

Using the same recruitment methods as in Study 1, 109 individuals participated in the study. Participants were mostly women (82.6\%), with an average age of 40.2 $(\mathrm{SD}=10.6)$. Less than half $(41.3 \%)$ reported having been given a diagnosis of depression in the past, $25.7 \%$ were currently taking antidepressants, and $17.4 \%$ were currently seeing a therapist.

\section{Materials}

Beck Depression Inventory-II (BDI; Beck et al. 1996), with item 9 removed as in Study 1, was used to measure the participants' level of depression. The average BDI score of the sample was 13.8 ( $\mathrm{SD}=12.1)$, ranging from 0 to 48 . That participants in Study 2 are less depressed than in Study 1 is likely due to the relative differences of recruitment channels: whereas in Study 1 most participants were recruited from the online forums and newsgroups, participants in Study 2 were mostly recruited from the per-pay study pool.

The Thinking about Decisions Questionnaire, developed for this study, was used to evaluate the participants' decisionmaking. The questionnaire was based on the same six 
scenarios used in Study 1, and was designed to prompt participants to think carefully about the decisions. For each scenario, participants were asked to make their decisions according to the steps cognitive-behavioral therapists (Beck 1995) and practitioners of Problem Solving Therapy (Nezu et al. 2007) often use to help clients with decisions, as follows:

1. Generate all options that might be realistic for a given scenario.

2. Rank-order the options, from their favorite (the one they would actually choose) to their least favorite. Satisfaction with their favorite choice was then rated on a 7-point Likert scale.

3. Think about their first and second option (most favorite and the runner-up), and list positive and negative features of one as compared to the other.

4. Report whether they would want to change their mind about their order of options, given this deliberation. Satisfaction with the final decision was reassessed on a 7-point Likert scale.

A scoring system for the free-response question was developed. Quality of participants' decisions was assessed by rating the options generated by the participants on the Productivity dimension (as in Study 1). The positive and negative features of the two favorite options were categorized into five "Sought" features and five "Avoided" features. The "Sought" group described the features of options that participants seemed to prefer or seek out, and contained five categories: Being proactive, Good outcome, Social benefits, Resources, and Feeling better. The "Avoided" group contained the features that participants seemed to dislike and eschew, and also consisted of five categories: Costs, Anxiety, Feeling bad (e.g., uncomfortable, or disappointed), Social problems, and Failure. When responses fit into more than one category, all applicable categories were noted.

Responses were coded by two independent Master's level raters (Y.L. and C.S.R.), blind to the participants' depression score or current treatment status. Both raters scored responses from 30 participants to evaluate the reliability of ratings. The ICCs for ratings of Productivity was 0.84 . The Cohen's kappa coefficients for the Sought subgroups ranged from 0.77 to 0.89 , and from 0.80 to 0.92 for Avoided subgroups.

Results

\section{Relationship to Depressive Symptoms}

There was no significant association between depressive symptoms and the number of options participants had generated $(r=0.06, P<.57)$.
Table 1 Regression models: BDI predicting decisional considerations

\begin{tabular}{lrrrrl}
\hline & $\beta$ & SE & \multicolumn{1}{l}{$t$} & DF & $P$-value \\
\hline Sought & & & & & \\
$\quad$ Good outcome & .02 & .01 & 1.65 & 107 & .10 \\
Proactive & .00 & .01 & .06 & 107 & .95 \\
Social benefits & -.01 & .01 & -.43 & 107 & .67 \\
Resources & .00 & .01 & .15 & 107 & .88 \\
Feel better & .01 & .01 & 1.15 & 107 & .25 \\
Avoided & & & & & \\
Costs & -.01 & .01 & -.78 & 107 & .44 \\
Anxiety & .04 & .01 & 4.57 & 107 & .0001 \\
Feeling bad & .00 & .01 & .39 & 107 & .72 \\
Social problems & .02 & .02 & .97 & 107 & .34 \\
Failure & .02 & .01 & 1.31 & 107 & .20 \\
\hline
\end{tabular}

With an exception of the desire to avoid anxiety $(\beta=.04, t(107)=4.57, P<0.0001$, still significant after controlling for relevant demographic and treatment variables), no other regression predicting decisional considerations (aspects of decisions that participants sought and those they were trying to avoid) from BDI was significant (Table 1). These findings suggest that decision goals do not vary based on the severity of depressive symptoms. However, BDI scores were associated with the reduced satisfaction with decisions, both with the initial decision and the final, post-deliberation decision $(r=0.26, P<0.01$, and $r=0.23, P<0.03$, respectively).

\section{Does Using Decision Tools Help Productivity of Decisions? Comparing the Results of Study 1 and Study 2}

Although the ranges of BDI scores were similar between the two studies, participants in Study 2 did not report as much depressive symptomatology, on average, as those in Study $1(t(232)=5.02, P<0.001, d=0.66)$. To make the results of the two studies comparable, we used a regression model as described below, to predict productivity of decisions from BDI scores and estimate unstandardized betas. Unstandardized betas are preferable because, unlike correlation coefficients: (1) they do not rely on the variance of the variables (which differed between the two studies) to estimate relationships, and (2) they rely on units of measurements as measured, rather than standardizing them, which makes comparisons of uneven distributions possible (i.e., 1 unit of BDI of distribution A is the same as 1 unit of BDI in distribution B).

The datasets from Study 1 and Study 2 were combined for a quasi-experimental joint analysis. To show the difference between the association of depression and productivity between the two studies, regression slopes of productivity as a function of BDI scores for the two studies 


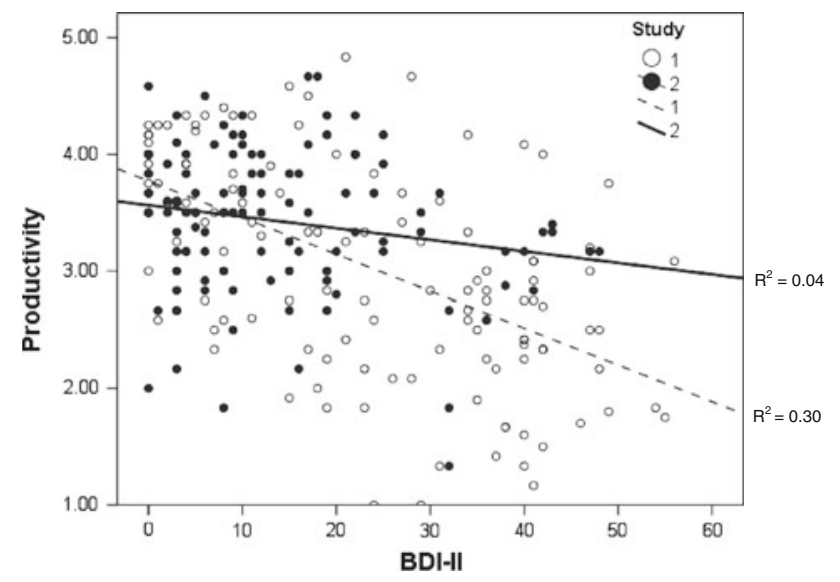

Fig. 1 Regression slopes of BDI scores predicting productivity, grouped by study

were compared. A significant interaction of the BDI scores and Study in predicting productivity would indicate a difference between the regression slopes obtained in Study 1 and that of Study 2. The results of a regression model indeed revealed a significant interaction of BDI scores and Study in predicting productivity, $t(230)=3.16, P<.002$, $R^{2}=.27, F(3,230)=28.19, P<.0001$ (Fig. 1). Simple slope analyses revealed that the slope was significant for the sample in Study $1(\beta=-.03, t(230)=-7.99$, $P<0.001$ ), but not significant for the sample in Study 2 $(\beta=-.01, t(230)=-1.78, P<.08)$. The unstandardized betas and the variance explained by BDI scores were considerably lower in Study 2 compared to Study 1 (Fig. 1). Including relevant demographic and treatment variables in the regression model did not alter the results.

\section{Discussion}

The results from Study 2 suggest that once individuals were prompted to use good decision-making strategies, few associations between decision-related variables and the depression scores were found. Of the decisional considerations indicated by participants, only one-desire to avoid anxiety - was found to be predicted by depressive symptoms. Depression scores likewise were not related to participants' ability to generate possible alternative behaviors for given decision situations. These results suggest that priorities and goals of depressed individuals, as well as their ability to use decision tools, may not differ from those of the nondepressed. Importantly, prompting the utilization of decision strategies seemed to improve the ability of depressed individuals to make productive decisions. Thus, of the two competing hypotheses discussed above, the findings are more consistent with the possibility that depressive deficits in decision making result from a failure to employ adaptive decision tools.
Associations between depression and satisfaction about decisions were also found. These findings are not surprising given the bias toward negative thinking, and the selective attention toward the negative, that have been documented in depression.

\section{General Discussion}

The two studies described in this report were designed to accomplish several goals. The first goal was to characterize the associations between the level of depressive symptomatology and decision-related attitudes and approaches. In addition to predictable differences, the findings suggested that individuals with greater levels of depressive symptoms seek out less information to help them with problem-solving and make use of fewer resources. They are also less likely to engage decisions that would resolve an ambiguity, which may be reflective of poor decision making skills, as well as perhaps of greater tolerance of ambiguous or uncertain situations. Second, a hypothesis that depression scores are associated with the goodness of decisions was tested. The results confirm an inverse relationship between depression and goodness of decisions, such that those that are more depressed make less productive decisions. This was corroborated by the inverse relationship found between depression scores and selfreported goodness of past outcomes, as well as the finding of an increased likelihood of using a previously failed strategy in a similar situation.

Third, the studies were meant to distinguish between two competing hypotheses: 1 . the relationship between depressive symptoms and goodness of decisions is due to poor decision-making skills, or 2. the said relationship is due to other factors, such as differences in goals or values. Findings from Study 2, in which we provided participants with a common set of decision-making tools, suggest that depressed individuals make poorer decisions because they fail to use adaptive decision-making strategies. Once good strategies were employed, the productivity of decisions of the more depressed participants converged with those of the less-depressed. Furthermore, there were no significant associations found between the participants' goals for decisions and their level of depression, with the exception of a desire to avoid anxiety. It is possible that wishing to eschew anxiety causes depressed individuals to avoid decisions that are potentially ambitious or risky. Based on the findings of Study 2, however, whatever influence anxiety might have on decision-making can be reversed by using good decision practices.

The results from these two studies offer support to the usefulness of some of the techniques used in CognitiveBehavioral Therapy (CBT) for depression. In assisting their 
clients with decisions, CBT practitioners encourage the use of tools such as Pros/Cons lists, examining several sides of the issue, and creatively generating options for action-all in the attempt to engage more thoughtful and productive decision-making (Beck 1995). A similar approach is used to address errors of judgment, such as jumping to conclusions. A therapist might encourage patients to think about issues more rationally and guide them through a more adaptive thought process. Problem Solving Therapy (PST; Nezu et al. 1989) is another therapeutic approach that addresses decision-making as a specific target of intervention. Research into CBT and PST demonstrated their efficacy vis-à-vis reduction of depressive symptoms; however, there is little research showing that techniques used in these treatments improve the actual decisions. Our findings suggest that those with higher depressive symptoms have specific deficits in decision-making and make poorer choices, and that these choices could be improved by the tools similar to those provided by the above treatments. A question future research may address is whether improvements in decision-making mediates treatment success in CBT or PST.

These studies have several limitations. Due to the reliance on convenience sampling, the studies differed in the average level of depression reported by participants. Furthermore, although the analysis in which data from the two studies were combined into one dataset treated the studies as two conditions in an experiment, it was quasi-experimental. Any conclusions drawn from the comparisons between the two samples should therefore be interpreted with caution. Although the raters were blind to the participant's depression status, and every effort was made to keep the ratings unbiased, aided by the manualized rating guides, it is possible that the depression status of participants could have been revealed through either the emotional tone of the response or through participants' inadvertent comments. However, this limitation would concern only the results of Study 1, with its significant relationships between depression scores and decisionrelated issues. Because the studies were conducted on the Web, depression levels were assessed via self-report, rather than via a clinical interview, and the presence of other symptoms or diagnoses that could affect decision-making was not assessed. Finally, the main variable of interestproductivity of a decision-reflects the degree of benefit a person might be expected to derive from making that decision in a hypothetical scenario. They therefore may only approximate the decisions participants might make in a real-life situation, or the benefit they might derive from that decision in a real-life situation.

Insofar as the choices a person makes throughout his or her life shape future outcomes, both in trivial matters and in those of considerable importance, the deficits in decision-making exhibited by depressed individuals are troubling. Bad decisions and the negative experiences resulting from them are likely to reinforce the already negative view depressed people hold of themselves and their abilities. Bad choices may also generate feelings of guilt for past failures, and support the feeling of hopelessness, as depressed individuals see themselves as being unable to make a positive impact on their future. Recognizing their difficulty with making decisions, they are likely to turn to avoidance of decisions as a coping strategy. This tendency is likely to lead to further missed opportunities, disappointments, and more negative outcomes overall. However, this work has shown that the ability to make sound decisions is not lost, as it can be brought back with a relatively simple manipulation, suggesting that teaching decision-making techniques in treatment and encouraging their use may be particularly productive for depressed individuals. Understanding the decision-making processes of depressed individuals and of those with other forms of psychopathology, can provide tools for clinicians, help people improve their lives, and enhance our understanding of the phenomenology of psychological disorders.

Open Access This article is distributed under the terms of the Creative Commons Attribution Noncommercial License which permits any noncommercial use, distribution, and reproduction in any medium, provided the original author(s) and source are credited.

\section{References}

Abramson, L. Y., Metalsky, G. I., \& Alloy, L. B. (1989). Hopelessness depression: A theory-based subtype of depression. Psychological Review, 96, 358-372.

Abramson, L. Y., Seligman, M. E., \& Teasdale, J. D. (1978). Learned helplessness in humans: Critique and reformulation. Journal of Abnormal Psychology, 87, 49-74.

APA. (2000). Diagnostic and statistical manual of mental disorders (4th edition, Text Revision). Washington, DC: Author.

Barber, J. P., \& DeRubeis, R. J. (1989). The ways of responding: A scale to assess compensatory skills taught in cognitive therapy. Behavioral Assessment, 14, 93-115.

Beck, J. S. (1995). Cognitive therapy: Basics and beyond. New York: Guilford.

Beck, A. T., Steer, R. A., \& Brown, G. K. (1996). BDI-II manual. San Antonio: The Psychological Corporation.

Bell, D. (1982). Regret in decision making under uncertainty. Operations Research, 30, 961-981.

Bell, D. E., Raiffa, H., \& Tversky, A. (Eds.). (1988). Decision making: Descriptive, normative, and prescriptive interactions. New York: Cambridge University.

Chambers, C. D., Johnson, K. A., Dick, L. M., Felix, R. J., \& Jones, K. L. (1996). Birth outcomes in pregnant women taking fluoxetine. New England Journal of Medicine, 335, 1010-1015.

Doerfler, L. A., Mullins, L. L., Griffin, N. J., Siegel, L. J., \& Richards, C. S. (1984). Problem-solving deficits in depressed children, adolescents, and adults. Cognitive Therapy and Research, 8, 489-499. 
Joffe, R. D., Dobson, K. S., Fine, S., Marriage, K., \& Haley, G. (1990). Social problem-solving in depressed, conduct-disordered, and normal adolescents. Journal of Abnormal Child Psychology, 18, 565-575.

Kahneman, D., Wakker, P., \& Sarin, R. (1997). Back to Bentham? Explorations of experienced utility. Quarterly Journal of Economics, 112, 375-406.

Klerman, G. L. (1980). Overview of affective disorders. In H. I. Kaplan, A. M. Freedman, \& B. J. Sadock (Eds.), Comprehensive textbook of psychiatry (3rd ed.). Baltimore: Willians \& Wilkins.

Kulin, N. A., Pastuszak, A., Sage, S. R., Schick-Boschetto, B., Spivey, G., Feldkamp, M., et al. (1998). Pregnancy outcome following maternal use of the new selective serotonin reuptake inhibitors: A prospective controlled multicenter study. Journal of the American Medical Association, 279, 609-610.

Leahy, R. L. (1999). Decision making and mania. Journal of Cognitive Psychotherapy, 13, 83-105.

Leahy, R. L. (2000). Mood and decision making: Implications for bipolar disorder. Behavior Therapist, 23, 62-63.

Leahy, R. L. (2001). Depressive decision making: Validation of the portfolio theory model. Journal of Cognitive Psychotherapy, 15, 341-362.

Leahy, R. L. (2002). Decision making and personality disorders. Journal of Cognitive Psychotherapy, 16, 209-225.

Loewenstein, G., O’Donoghue, T., \& Rabin, M. (2003). Projection bias in predicting future utility. Quarterly Journal of Economics, $118,1209-1248$.

Loomes, G., \& Sudgen, R. (1982). Regret theory: An alternative theory of rational choice under uncertainty. The Economic Journal, 92, 805-824.

Marx, E. M., Williams, J. M. G., \& Claridge, G. C. (1992). Depression and social problem solving. Journal of Abnormal Psychology, 101, 78-86.

Monroe, M. R., Skowronski, J. J., MacDonald, W., \& Wood, S. E. (2005). The mildly depressed experience more post-decisional regret than the non-depressed. Journal of Social and Clinical Psychology, 24, 665-690.
Murphy, F. C., Rubinsztein, J. S., Michael, A., Rogers, R. D., Robbins, T. W., Paykel, E. S., et al. (2001). Decision-making cognition in mania and depression. Psychological Medicine, 31, 679-693.

Nezu, A. M., Nezu, C. M., \& D'Zurilla, T. J. (2007). Solving life's problems: A 5-step guide to enhanced well-being. New York: Springer Publishing Co.

Nezu, A. M., Nezu, C. M., \& Perri, M. G. (1989). Problem solving therapy for depression: Theory, research and clinical guidelines. New York: Wiley.

Nezu, A. M., \& Perri, M. G. (1989). Social problem-solving therapy for unipolar depression: An initial dismantling investigation. Journal of Consulting and Clinical Psychology, 57, 408-413.

Platt, J. J., \& Spivack, G. (1975). Manual for the means-ends problem-solving procedure (MEPS): A measure of interpersonal cognitive problem-solving skill. Philadelphia: Hanhemann Community Mental Health/Mental Retardation Center. (Unpublished document).

Ritov, I., \& Baron, J. (1995). Outcome knowledge, regret, and omission bias. Organizational Behavior and Human Decision Processes, 64, 119-127.

Schwartz, B., Ward, A., Monterosso, J., Lyubomirsky, S., White, K., \& Lehman, D. R. (2002). Maximizing versus satisficing: Happiness is a matter of choice. Journal of Personality and Social Psychology, 83, 1178-1197.

Suri, R., Altshuler, L. A., \& Mintz, J. (2004). Depression and the decision to abort. American Journal of Psychiatry, 161, 1502.

Wierzbicki, M. (1984). Social skills deficits and subsequent depressed mood in students. Personality and Social Psychology Bulletin, 10,605 .

Young, H. F., \& Bentall, R. P. (1995). Hypothesis testing in patients with persecutory delusions: Comparison with depressed and normal subjects. British Journal of Clinical Psychology, 34(Pt 3), 353-369. 